\title{
Attention demands of visual search
}

\author{
GORDON D. LOGAN \\ Erindale College, University of Toronto, Mississauga, Ontario, Canada LSL 1C6
}

\begin{abstract}
The relation between attention demand and the number of items in the array (array size) was investigated by engaging subjects in a primary search task and measuring spare capacity at different points in time, with a secondary tone task that occurred randomly on half of the trials. The major variables in both tasks were array size $(4,8$, or 12 letters) and stimulus onset asynchrony (SOA: $-400,-200,0,200,400$, and $600 \mathrm{msec}$ ). Subjects were able to perform the tasks quite independently, and most of the interference that resulted from nonindependence appeared in tone-task performance. The amount of interference (i.e., maximum tone reaction time) was independent of array size, but the duration of interference (i.e., the number of SOAs at which tone reaction time was elevated) increased with array size. The findings were interpreted as supporting unlimited-capacity models of visual search performance.
\end{abstract}

When a person's attention is engaged in searching for a target in a visual array, to what extent are the attention demands of searching determined by the number of items in the array (array size)? This question has stimulated thought and research for several years (for a review, see Smith \& Spoehr, 1974), yet remains unanswered. The earlier efforts have made it clear, however, that more is required than a simple account of the observed increase in reaction time and error rate with array size. Assuming that attention demands increase with array size can lead one to predict the same increase in reaction time and error rate as assuming they do not. Moreover, assuming the items are processed simultaneously, in parallel, can lead to the same predicted array-size effect as assuming items are processed one at a time, in series. These points have been demonstrated formally by Taylor (1976) and Townsend (1974), leaving the search literature in a state of seemingly permanent uncertainty:

The present paper adopted a different strategy for measuring attention demands, motivated by technical advances in the attention literature. The idea was to vary attention demands and array size independently by engaging people in a demanding concurrent task while searching. The relation between attention demand and array size may then be revealed by changes in the array-size effect in search performance corresponding to changes in the attention demands of the concurrent task, or by the effect of array size itself on performance in the concurrent task, or both. If larger arrays do demand more attention, they should suffer

This research was supported in part by Grant AO 127 from the National Research Council of Canada to Albert S. Bregman at McGill University. I would like to thank Jane Zbrodoff, Alan Quapick, Morris Moscovitch, and Bruce Schneider for comments on the manuscript. Requests for reprints may be addressed to Gordon D. Logan, Department of Psychology, Erindale College, University of Toronto, Mississauga, Ontario, Canada LSL 1 C6. more interference from concurrent activity and interfere more with the concurrent task (for a full development of this argument, see Logan, 1978). Using this technique, predictions may be developed to distinguish the four classes of models that predict identical array-size effects.

Interference between tasks will be stronger the more the demands on attentional capacity exceed the supply. Since people are flexible in their allocation of capacity, able to change it in response to instructions or expected value (e.g., Shulman \& Fisher, 1972), it is convenient to designate one of the tasks as primary, so that it always receives sufficient attention for optimal performance, and the other as secondary, so that it may only draw on spare capacity left over from the primary task (Kahneman, 1973; Posner \& Boies, 1971). Under these conditions, the effects of concurrent activity are most prominent in the secondary task. Moreover, predictions derived with search as the secondary task should converge on predictions derived with search as the primary task.

First, consider changes in the array-size effect when search is the secondary task. Limited-capacity parallel models must predict a larger array-size effect with concurrent activity than without. They rest on the assumption that all items are processed simultaneously and draw on attentional capacity simultaneously, so that attention demands increase with array size. Assuming further that the speed and accuracy of processing the target are reduced in proportion to the load on capacity, these models predict the observed array-size effect (Atkinson, Holmgren, \& Juola, 1969; Nickerson, 1972; Rumelhart, 1970). When capacity is consumed by concurrent activity, proportionately less capacity is available for processing each item, so reaction time and error rate should increase further in proportion to array size. This prediction is illustrated graphically in Figure $1 \mathrm{~A}$.

Unlimited-capacity parallel models predict no change in the array-size effect with concurrent activity. Items 

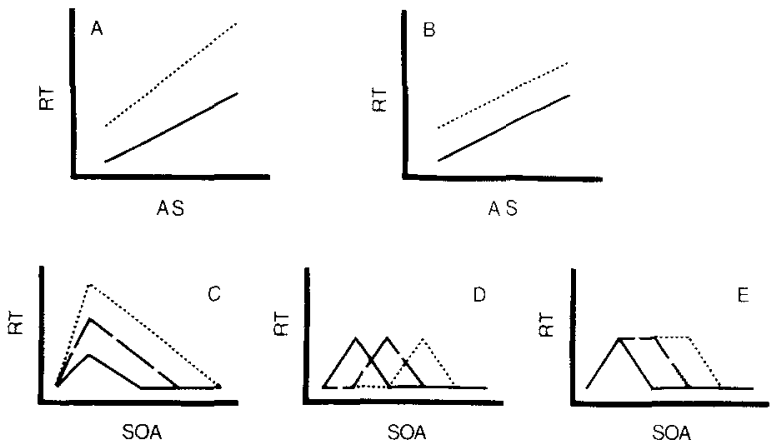

Figure 1. Possible outcomes in concurrent-task search experiments. (Panels A and B represent search-task data when search is the secondary task. Reaction time and array size are the coordinates; the top lines represent concurrent-activity trials, the bottom lines, single-task trials. In A, attention demands increase with array size; in $B$, attention demands are constant for all array sizes. Panels $C, D$, and $E$ represent secondary-task data when search is the primary task. Reaction time and stimulus onset asynchrony are the coordinates; solid lines $=4$ Hetter arrays; broken lines $=8$ - tetter arrays; dotted lines $=12$-letter arrays. The contours need not peak at a point nor increase and decrease linearly around the peak; these aspects of Panels $\mathrm{C}$, $D$, and $E$ are for graphic convenience. The models predict only monotonic increases and decreases that are gradual rather than abrupt to account for statistical variation in the duration of attention demand. In $\mathbf{C}$, the amount and duration of attention demand increases with array size; in $\mathrm{D}$, the amount and duration of attention demand are constant for all array sizes; in $E$, the amount of attention demand is constant, but the duration increases with array size.)

are assumed to be processed simultaneously, but without drawing attentional capacity. Each item is thought to add noise to a decision process, and since speed and accuracy of processing the target are reduced in proportion to the total amount of noise, the array-size effect is predicted (Estes, 1972; Gardner, 1973; Shiffrin \& Geisler, 1973). Since the processes underlying the array-size effect do not demand attention, concurrent activity cannot change the array-size effect, although it may increase reaction time by a constant amount for all array sizes, as illustrated in Figure 1B.

Serial models predict the array-size effect by assuming that items are processed one at a time, so that processing time (and thus reaction time) must increase with array size. Limited-capacity serial models assume that processing each item requires attention (e.g., Shiffrin \& Schneider, 1977), so that attention demands increase with array size. When available capacity is reduced by concurrent activity, each item will require more time to be processed, so that the array-size effect will become stronger, as in Figure 1A.

Unlimited-capacity serial models assume that processing each item does not require attention; attention demands should be constant for all array sizes, so the array-size effect should not change with concurrent activity, as in Figure 1B. However, serial processing must require a certain amount of bookkeeping, at least the system must determine when it has finished processing the current item and which item to process next, and it is possible that this bookkeeping requires attention.

With this assumption, unlimited-capacity serial models would predict a larger array-size effect with concurrent activity, as in Figure 1A, since the number of bookkeeping operations would increase with array size. Note that this assumption does not change the predictions of the other models: In parallel models, the amount of bookkeeping required is independent of array size, so the predicted effects would not change. Serial limited-capacity models already predict a larger array-size effect with concurrent activity; bookkeeping would enhance the effect, but the data should still look like Figure 1A.

I have reported a series of experiments in which visual search was the secondary task, performed in the retention interval of a primary short-term memory task (Logan, 1976, 1978). In all experiments, a concurrent memory load increased reaction time, but the array-size effect remained unchanged; the results resembled Figure 1B (also see Logan, 1976, Experiment 2). These findings reduce the field of available models, supporting unlimited-capacity parallel models and the unlimited-capacity serial models that assume no bookkeeping (see Table 1).

The present study was designed to reduce the field further. Visual search was the primary task, so that the attention demands associated with array size would be revealed most prominently in secondary-task performance. People were engaged in a visual search task in which they determined whether an array of 4,8 , or 12 letters contained an A or a V. At the same time, they performed a secondary, simple reaction time task, responding to a tone presented at one of six stimulus onset asynchronies (SOAs) defined relative to the onset of the array. Under these conditions, the function relating secondary-task performance to SOA defines, for the primary task, a "contour" of attention demand over time, which may be used to compare momentary demands of the same task at different points in time, or to compare the demands of different versions of the primary task. This procedure and rationale was first used by Posner and Boies (1971)

Table 1

Predictions with Search as the Primary Task and the Secondary Task

\begin{tabular}{lllllll} 
& \multicolumn{5}{c}{ Possible Outcomes } \\
\cline { 2 - 6 } \multicolumn{1}{c}{ Assumptions } & A+C & A+D & A+E & B+C & B+D & B+E \\
\hline No Bookkeeping & LP & & LS & & UP;US & \\
Bookkeeping & LP;LS;US & & LS;US & & & UP
\end{tabular}

Note-Columns represent all possible combinations of outcomes in primary-and secondary-task search data. $A, B, C, D$, and $E$ refer to outcomes illustrated in Figure 1. The entries represent the models predicting the outcomes; $L=$ limited capacity; $U=$ unimited capacity; $S=$ serial; $P=$ parallel. 
and has since gained wide currency (e.g., Comstock, 1973, 1975; Ells, 1973; Millar, 1975; Posner \& Klein, 1973; Proctor \& Fisicaro, 1977; Shwartz, 1976).

In the present study, the range of SOA extended from -400 to $600 \mathrm{msec}$. Pilot data had suggested that this range would capture the onset and offset of attention to the search task, so that a complete contour of attention demand could be obtained for each array size. Two aspects of the contour, its height and breadth, are important, as they represent, respectively, the magnitude and duration of the attention demands of searching.

Again, predictions may be developed for each of the four models: Limited-capacity parallel models predict contours that are higher and broader the larger the array, reflecting, respectively, the greater momentary demands of the larger arrays and the longer time spent in active attention to the array to meet the greater demand. The predicted contours are illustrated in Figure 1C.

Unlimited-capacity parallel models predict contours equal in height and breadth for each array size, since the attention demands of searching do not increase with array size and search need not require attention until the target has been determined, after which the attention-demanding processes should be equal in duration for each array size. Because it will take more time to determine the target in a large array, search will begin to demand attention later, and the peak of the contour should appear later in time, further to the right on the SOA axis. These contours are illustrated in Figure 1D. It is possible, however, that the onset of the array might evoke attention-demanding processes other than those designed to determine which target was presented (i.e., the bookkeeping processes mentioned earlier). Perhaps the normal response tendencies of the visual system must be changed temporarily to best discriminate the targets; the maintenance of such a set might constitute attentiondemanding bookkeeping (Logan, 1978). Similarly, the response tendencies of the hands might be altered dynamically (since people do not ordinarily press buttons when they see letters), and the maintenance of the new set might require capacity (Klapp, 1976). These processes would begin to demand attention once the array appeared and would continue to do so until a response was made. The time to respond depends on the duration of the unlimited-capacity target-determining process which, in turn, depends on array size. Thus, the duration of attention demand would necessarily increase with array size, although the amount demanded would not. The predicted contours, assuming bookkeeping, appear in Figure 1E. Note that the bookkeeping assumption does not change the predictions of limitedcapacity parallel models, since they already predict that the duration of attention demand should increase with array size.

Limited-capacity serial models predict contours equal in height, with breadth increasing with array size because momentary demands are constant across array size (since only one item is processed at a time), yet more time must be spent attending to the array (processing items) the larger the array. The predicted contours are illustrated in Figure 1E. The predictions may or may not be changed by assuming bookkeeping, depending on the nature of the assumed bookkeeping. Minimally, the system must be able to determine when it has finished processing the current item (so the next item can be selected) and to select the next one. Serial scanning theory holds that scanning is efficient in that items are only processed once (see Nickerson, 1972; Sternberg, 1975), and this imposes further constraints on bookkeeping. The items selected may be kept track of dynamically, perhaps by remembering the ones that have been selected (or equivalently, the ones that have not). In this case, momentary attention demands would increase with array size, and performance would resemble Figure 1C. Alternatively, the data structure in which the items are held might be exploited so that it keeps track of the items that have and have not been selected; selection might be based on some local property of the data structure that permits exhaustive sampling without replacement. For example, a circular array of letters has a ring-like structure that may be sampled exhaustively by iteratively selecting the next item clockwise from the current one. In this case, the physical array provides the organization-the spatial structure of the world constrains the activity of the perceptual system-and attention demands would be constant across array size. Performance would resemble Figure 1E.

Unlimited-capacity serial models predict contours equal in height and breadth, as in Figure 1D, since neither the amount nor the duration of attention demand vary with array size. Assuming bookkeeping that concerns only the current item and the next one to be processed, performance would resemble Figure 1E. Assuming bookkeeping that concerns all unprocessed items, performance would resemble Figure $1 \mathrm{C}$.

It is important to consider these predictions of secondary-task performance when search is the primary task in combination with the earlier predictions of search performance when search was the secondary task. The two sets of predictions converge on the models and their assumptions; models may stand or fall on their ability to predict performance in both situations. The predicted combinations are summarized in Table 1 , where columns represent possible combinations of outcomes with search as the primary and the secondary task, the rows indicate whether or not bookkeeping is assumed, and the entries represent the models that predict the outcomes given the assumptions. Previous data with search as the secondary task have shown that the array-size effect does not change with concurrent activity, as in Figure 1B (Logan, 1976, 1978). This restricts the set of possible models to unlimited-capacity serial models that assume no bookkeeping and 
unlimited-capacity parallel models that may or may not assume bookkeeping. From Table 1, the possible outcomes in the present experiment where search is the primary task are (1) contours equal in height and breadth for all array sizes (Figure 1D), supporting unlimited-capacity serial and parallel models that assume no bookkeeping, and (2) contours equal in height with breadth increasing with array size (Figure 1E), supporting unlimited-capacity parallel models that assume bookkeeping. The first outcome would answer the question that began this paper; it would indicate that attention demands do not increase with array size. The second outcome would support one model uniquely and suggest the importance of bookkeeping processes.

Finally, the procedure allowed an estimate of the effects of concurrent activity on search performance. Since the tone for the concurrent task was presented on only half of the trials, the difference between search performance on tone and no-tone trials may also reveal the attention demands of searching. If it is, indeed, appropriate to apply the previous results with search as the secondary task to the present situation, one should expect that responding to the tone would not increase the array-size effect. This prediction is, of course, weakened because search was the primary task. Nevertheless, a larger array-size effect with concurrent activity would be most informative.

\section{METHOD}

\section{Subjects}

Twelve graduate and undergraduate students and laboratory staff from McGill University served as subjects. Four were male and eight were female. None reported any perceptual defect, visual or auditory, and each was paid for participating in four 1-h sessions.

\section{Apparatus and Stimuli}

The visual stimuli were arrays containing 4,8 , or 12 different letters equally spaced around an imaginary circle centered on the fixation point (see Logan, 1978, Figure 5). Each array contained one target letter, an A or a V. Each array size was represented by 48 different arrays in which each target letter appeared in each position equally often. The same was true for each nontarget letter (all remaining letters except $Q$ ), within sampling limitations. The arrays were made from black uppercase Letraset (717) mounted on white cards. The exposure of the array was preceded and followed by a fixation field containing a small black dot in the center of a white field.

The stimuli were exposed in a Gerbrands three-field tachistoscope (Model T-3B-1) with a viewing distance of $80 \mathrm{~cm}$. At this distance, each letter subtended about 26 by $26 \mathrm{~min}$ of visual angle, and the diameter of the imaginary circle on which the letters were placed subtended about $4 \mathrm{deg}$ of visual angle. The luminance of fixation and array fields was matched at $8 \mathrm{fL}$; during testing the room was dimly lit by a $40-\mathrm{W}$ bulb. Each day, $5 \mathrm{~min}$ were allowed for dark adaptation before testing began.

The auditory stimulus was a $1,000-\mathrm{Hz}$ sine tone produced by an Electronic Institute tone generator (Model 377) and presented binaurally through headphones (Koss pro/4AA) at a comfortable listening level. The events on a trial began with the closure of a switch, which initiated both the timer associated with the tachistoscope and a set of Hunter timers (Model 111-C,
Series D) that controlled SOA and the duration of the tone. The array appeared in the tachistoscope $400 \mathrm{msec}$ after the switch closed. It remained on for $600 \mathrm{msec}$. The tone was presented for $500 \mathrm{msec}$ at one of six SOAs, which ranged from -400 to $600 \mathrm{msec}$ in $200-\mathrm{msec}$ steps (positive values of SOA indicate that the tone followed the onset of the array).

Thus, the onset of the earliest tone immediately followed the closure of the switch, and the onset of the latest tone coincided with the termination of the array.

The tone was presented on half of the trials. Trial-to-trial variation in SOA required that the Hunter timers controlling SOA be reset before each trial. Because the adjustment was audible to the subjects, the timers were also reset before trials on which the tone was withheld. On those trials, the circuit from the tone generator to the headphones was broken by the operation of a silent switch.

In the search task, reaction time was measured from the onset of the array, using a digital timer. The timer began with the onset of the array, and stopped when the subject pressed one of two buttons mounted on a panel placed in front of him or her. Pressing each button also illuminated a separate light so that response accuracy could be monitored. In the tone task, reaction time was measured from the onset of the tone by a similar digital timer that started with the onset of the tone and stopped when the subject pressed a third button. All reaction times were measured in milliseconds.

\section{Procedure}

Each trial began with a verbal ready signal from the experimenter ("ready?"), to which the subject responded "yes" if he or she had the fixation point in sharp focus. About $.5 \mathrm{sec}$ later, the experimenter closed the switch that initiated the events of the trial in the manner described above. Since the subjects could hear the click of the switch, it served as a warning signal, occurring reliably $400 \mathrm{msec}$ before the onset of the array.

Fach day, each subject completed 144 trials in four blocks of 36 trials. Each array size and target letter occurred equally often in each block. A tone occurred on half of the trials in each block, such that each SOA was paired once per block with each array size. Within these constraints, the order of conditions was randomized. One order of 144 trials was constructed, and it and its inverse were used throughout the experiment. Each subject alternated between the two orders day by day, half of the subjects beginning with one order (i.e., ABAB), half beginning with the other (i.e., BABA).

Subjects were told to rest the middle and index fingers of their right hands on the two response buttons for the search task. They were told to press the left button with their index finger if the array contained an $\mathrm{A}$, and the right button with their middle finger if it contained a V. To the left of these two buttons was the response button for the tone task. Subjects were told to rest the index finger of their left hand upon it, and to press it immediately when they heard a tone.

Before testing began, subjects saw and heard examples of the stimuli, and had the events on a typical trial described to them in detail. The search task was defined as primary in that (1) after each trial subjects were told their reaction time and accuracy in the search task, but not in the tone task, (2) the search task occurred reliably on every trial, while the tone task occurred randomly on half of the trials, and (3) subjects were told that the search task was the more important of the two, and that they should concentrate on it, responding as quickly and accurately as possible. They were told to respond quickly in the tone task as well, but speed was not strongly emphasized as it was in the search task. The subjects were told to respond to the tasks as independently as possible, and in particular, to avoid response-grouping strategies such as responding to the two tasks simultaneously on each trial. Each subject completed four 1-h sessions on successive days. No more than 2 days 
elapsed between consecutive sessions. After the first session, the instructions were reviewed briefly during the dark-adaptation period to insure that the subjects still remembered them.

\section{Data Analysis}

Each day, each subject completed four concurrent-task trials at each combination of array size and SOA. The mean reaction time in each combination was computed for each subject for both the search task and the tone task. Each day, each subject completed 24 single-task trials at each array size in the search task, and mean reaction times were computed there as well. The first session was considered practice, and only data from the last three sessions were analyzed. Since previous studies with the same stimuli had shown that most of the improvement with practice occurred between the first and second sessions, each subject's data were averaged over the last three sessions, and these scores were submitted to analysis of variance.

\section{RESULTS}

\section{The Tone Task}

Mean reaction times across subjects and days are displayed in Figure 2. Each point in the figure is based on 144 observations. Panel A presents the data plotted conventionally as a function of the time at which the tone was presented (SOA), whereas Panel B represents the same data plotted as a function of the time at which the response to the tone occurred. The two plots are presented because each misrepresents the temporal relation between maximal interference and the onset of the array. Panel A suggests that maximal interference occurred before the onset of the array; Panel B suggests it occurred around the termination of the array, about the same time as a response occurred in the search task (vertical lines). This ambiguity arises because the measure of interference is itself an interval of time, extending from the onset of the tone to the completion of the buttonpress response. Interference cannot be represented as a point on the SOA axis because it itself extends along the SOA axis. Thus, corresponding points in the two plots represent the ends of intervals during which the tone task was subject to interference. It is clear from the two plots that the tone responses showing maximal interference overlapped in time with the

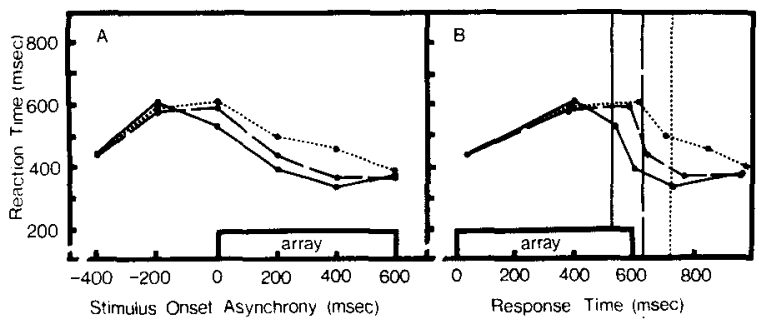

Figure 2. Mean reaction time in the tone task as a function of stimulus onset asynchrony (Panel A) and the time at which the response to the tone occurred (Panel B). (Array size is the parameter; solid lines $=4$-letter arrays; broken lines $=8$-letter arrays; dotted lines $=12$ tetter arrays. The vertical lines in Panel B represent mean search reaction times, across SOA, for trials on which the tone was presented.) responses to the array. Responses to tones presented $200 \mathrm{msec}$ before the array finished some $400 \mathrm{msec}$ after the array began. Even with no interference, the fastest possible response to these tones (estimated from the fastest observed reaction time) would have finished some $120 \mathrm{msec}$ after the array began. In either case, there is sufficient temporal overlap in the responses to the tasks to have produced dual-task interference; one can feel confident that tone-task interference resulted from processing the array.

The temporal relation between secondary-task interference and primary-task events has not been ambiguous in previous studies because the intervals between primary-task events have been large relative to secondary-task reaction times (e.g., Posner \& Boies, 1971). Thus, either plot would lead to the same conclusions.

Inspecting Panel A, it is clear that performance was strongly influenced by SOA $[F(5,55)=30.131$, $\mathrm{p}<.001]$. Reaction time increased from the $-400-\mathrm{msec}$ SOA to the $-200-\mathrm{msec}$ SOA, reached a peak between SOAs of -200 and $0 \mathrm{msec}$, and declined thereafter. The contours for each array size differed systematically from each other, as indicated by the significant effect of array size $[\mathrm{F}(2,22)=14.214, \mathrm{p}<.001]$ and its interaction with SOA $[\mathrm{F}(10,110)=5.242, \mathrm{p}<.001]$ The contours for each array size rose together and reached a peak at the same height (maximum reaction times were 607,595 , and $609 \mathrm{msec}$ for 4-, 8-, and 12-letter arrays, respectively), but declined separately such that the breadth of contour increased with array size. This suggests that the amount of attention demanded by the search task was independent of array size, but the duration of attention demand was not. It tended to increase with array size.

Errors were relatively rare in the tone task (mean proportion $=.021$ ), and virtually all of them were misses. The proportion of errors for each array size and SOA appear in Table 2.

\section{The Search Task}

Mean reaction times from concurrent-task trials are plotted as a function of SOA in Figure 3. Each point on the function for each array size is based on 144 observations. Single points to the right of the functions represent mean reaction times from no-tone trials. Each of these points is based on 864 observations.

Performance in the search task was strongly affected by array size $[F(2,22)=60.289, p<.001]$. Reaction times increased monotonically with array size at each SOA, and in the no-tone trials. However, SOA and the interaction between SOA and array size also had significant effects $[F(5,55)=7.292, \quad p<.001$, and $\mathrm{F}(10,110)=2.937, \mathrm{p}<.003$, respectively]. This is disturbing, since the interpretation of secondary-task data is clearest when the secondary task has no effect on primary-task performance (Kantowitz, 1974). But 
Table 2

The Proportion of Errors in the Tone Task, the Interresponse Intervals in Milliseconds, and the Proportion of Errors in the Search Task, Each as a Function of SOA and Array Size

\begin{tabular}{|c|c|c|c|c|c|c|c|}
\hline \multirow[b]{2}{*}{ Measure } & \multirow{2}{*}{$\begin{array}{c}\text { Array } \\
\text { Size }\end{array}$} & \multicolumn{6}{|c|}{ Stimulus Onset Asynchrony } \\
\hline & & -400 & -200 & 0 & 200 & 400 & 600 \\
\hline Errors (Tone Task) & $\begin{array}{r}4 \\
8 \\
12\end{array}$ & $\begin{array}{l}.007 \\
.007 \\
.000\end{array}$ & $\begin{array}{l}.007 \\
.014 \\
.007\end{array}$ & $\begin{array}{l}.014 \\
.007 \\
.014\end{array}$ & $\begin{array}{l}.007 \\
.035 \\
.035\end{array}$ & $\begin{array}{l}.035 \\
.063 \\
.028\end{array}$ & $\begin{array}{l}.021 \\
.042 \\
.035\end{array}$ \\
\hline Interresponse Interval & $\begin{array}{r}4 \\
8 \\
12\end{array}$ & $\begin{array}{l}-488 \\
-544 \\
-619\end{array}$ & $\begin{array}{l}-163 \\
-287 \\
-364\end{array}$ & $\begin{array}{l}-27 \\
-81 \\
-110\end{array}$ & $\begin{array}{l}90 \\
35 \\
13\end{array}$ & $\begin{array}{l}223 \\
167 \\
128\end{array}$ & $\begin{array}{l}476 \\
338 \\
322\end{array}$ \\
\hline Errors (Search Task) & $\begin{array}{r}4 \\
8 \\
12\end{array}$ & $\begin{array}{l}.104 \\
.083 \\
.167\end{array}$ & $\begin{array}{l}.049 \\
.076 \\
.188\end{array}$ & $\begin{array}{l}.021 \\
.104 \\
.146\end{array}$ & $\begin{array}{l}.014 \\
.063 \\
.118\end{array}$ & $\begin{array}{l}.049 \\
.049 \\
.118\end{array}$ & $\begin{array}{l}.049 \\
.035 \\
.076\end{array}$ \\
\hline
\end{tabular}

since the SOA effects on search performance were neither large nor regular enough to yield a consistent interpretation, it may be most reasonable to regard them as noise.

This interpretation is supported somewhat by the overall similarity of performance on tone and no-tone trials. For tone trials, the mean reaction times (across SOA) for 4-, 8-, and 12-letter arrays were 532, 627, and $701 \mathrm{msec}$, respectively. The corresponding means from no-tone trials were 524,626 , and $718 \mathrm{msec}$. At no SOA was the array-size effect larger than the one observed on no-tone trials. Thus, there is no evidence that performing the tone task while searching increased search reaction time relative to no-tone trials by an amount proportional to array size.

The proportion of errors for tone trials in the search task appear in Table 2 , representing each combination of array size and SOA conditions. The proportion of errors for no-tone search trials was $.057, .056$, and .119 for 4-, 8-, and 12-letter arrays, respectively.

\section{Tone and Search Tasks Together}

Subjects were instructed to perform the two tasks independently, and if they could not, to protect the primary search task so that the ensuing interference

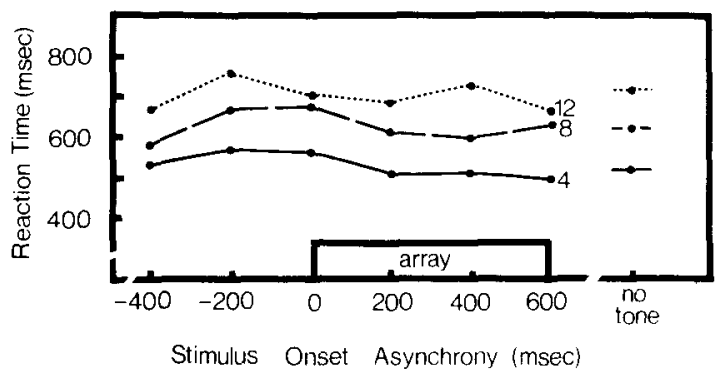

Figure 3. Mean search reaction time for trials on which a tone was presented as a function of stimulus onset asynchrony. (Array size is the parameter; solid lines $=4$-letter arrays; broken lines $=8$-letter arrays; dotted lines $=12$-letter arrays. Single points to the right of each function represent the corresponding mean reaction times from search trials on which the tone was withheld.) would only be apparent in performance on the secondary tone task. The significant main effect of SOA in the analysis of the tone data suggests that subjects were not able to handle the tasks independently, and the significant main effect of SOA and its interaction with array size in the search data suggests that they were not able to restrict the ensuing interference to the secondary task. The purpose of this section is to report measures of the relative strength of these effects. This may be done in two ways: (1) by comparing variance components associated with main effects and interactions in the two separate analyses of the tasks, and (2) by examining trends and variance components in an analysis of the interval between responses to the two tasks (interresponse interval, hereafter, IRI).

Variance components. The variance components associated with each main effect and interaction in the two tasks were computed following the method of Vaughan and Corballis (1969) and appear in Table 3.

If subjects had been able to restrict concurrent-task interference to tone-task performance, the variance components associated with SOA and with the interaction between SOA and array size should be substantial in the tone task but negligible in the search task. From the estimates in Table 3, it is clear that this was the case. The variance component associated with SOA was about 12 times larger in the tone task than in the search task, and the component associated with the interaction was about twice as large. It would appear that, for the most part, subjects were able to protect the search task as instructed.

Interresponse intervals. Following Kahneman (1973), IRIs were computed by applying the formula, $\mathrm{IRI}=$ Tone RT + SOA - Search RT, to the mean reaction times in each combination of array size and SOA conditions for each subject. The means across subjects appear in Table 2 , and the variance components from an analysis of variance on the IRI data appear in Table 3 (both main effects and the interaction were significant, $p<.003$ ).

If subjects had been able to perform the two tasks independently, IRI should be a linear function of SOA 
Table 3

Variance Components of the Effects of Array Size (AS) SOA, and Their Interaction in the Tone Task, the Search Task, and the IRI Analysis

\begin{tabular}{lrrr}
\hline & \multicolumn{3}{c}{ Effect } \\
\cline { 2 - 4 } \multicolumn{1}{c}{ Analysis } & \multicolumn{1}{c}{ AS } & \multicolumn{1}{c}{ SOA } & AS x SOA \\
\hline Search Task & $4,724.824$ & 591.341 & 199.835 \\
Tone Task & 386.456 & $7,030.866$ & 411.264 \\
Interresponse Interval & $2,552.970$ & $90,097.817$ & 277.264 \\
\hline
\end{tabular}

with a slope of one (i.e., adding $100 \mathrm{msec}$ to SOA would add $100 \mathrm{msec}$ to IRI). If, on the other hand, subjects could not perform the two tasks together, IRI should be constant, independent of SOA, particularly at short SOAs where interference is typically strongest (see Kantowitz, 1974). If either response was lengthened at the shorter SOAs, the function relating IRI to SOA would flatten. Indeed, if one response were to become completely refractory, the (local) slope of the function would become zero. Since the range of SOA in the present experiment captured the beginning and end of interference, this local flattening should appear in the middle of the function, with the ends linear with unit slope. Thus, independent performance on the two tasks would be seen as a linear trend in the IRI data, whereas interference or dependent performance would be seen as a cubic trend (for a similar argument, see Kahneman, 1973, Chapter 9).

Only the linear and cubic trends were significant in the analysis $[\mathrm{F}(1,55)=517.944, \mathrm{p}<.001$, and $F(1,55)=6.164, p<.050$, respectively]. The linear was the stronger of the two, accounting for $98 \%$ of the variance component due to SOA, while the cubic trend accounted for $1 \%$. It appears that, although subjects did experience some interference when performing the two tasks concurrently, they were able to perform them quite independently.

\section{DISCUSSION}

This paper began by asking to what extent the attention demands of searching are determined by array size. An answer to this question was obtained by having people respond to tones presented randomly at different SOAs while they were engaged in searching arrays of different sizes for the presence of an $\mathrm{A}$ or a $\mathrm{V}$. The data suggest that the people were able to perform the two tasks independently for the most part, and that they were able to restrict most of the interference resulting from concurrent performance to the secondary tone task. The small amount of interference observed in the primary search task was not easily interpretable and perhaps should be regarded as noise. Given these findings, the functions relating tone reaction time to SOA may be interpreted as measures of the amount and duration of the attention demands of searching.
The data (see Figure 2) clearly indicate that the amount of attention demanded was the same for each array size, but the duration of attention demand increased with array size. Thus, the initial question is answered.

The answer, combined with previous data obtained with search as the secondary task, may reduce the field of possible models to one, the unlimited-capacity parallel models that assume bookkeeping. By themselves, the data from the present tone task are consistent with (1) limited-capacity serial models with and without bookkeeping assumptions, (2) unlimitedcapacity serial models with bookkeeping assumptions, and (3) unlimited-capacity parallel models with bookkeeping assumptions. These models predict the observed constant attention demands, which were longer in duration with larger arrays. By themselves, the previous data with search as the secondary task (Logan, 1976, 1978), and indeed, the present search data support (1) unlimited-capacity serial models without bookkeeping assumptions and (2) unlimitedcapacity parallel models with and without bookkeeping assumptions. These models predict the observed constancy of the array-size effect under varying conditions of attention. The two sets of data together are more consistent with the unlimited-capacity parallel models that assume bookkeeping than with any other model, as only they predicted observed combination of outcomes (see Table 1).

The possible nature of bookkeeping processes invites speculation. In serial models, some bookkeeping processes are obvious--those keeping track of the serial comparison process. In the favored parallel models, bookkeeping might involve maintaining a set to discriminate the targets (Logan, 1976, 1978) and maintaining the appropriate responses in a state of readiness (Klapp, 1976). More generally, one might expect that the momentary organization and coordination of cognitive resources sufficient to perform the task would demand attention. Many of the resources called upon in laboratory tasks have been practiced extensively in other contexts and might function automatically, at least in familiar contexts. What is new in the laboratory situation is the constellation of resources required for performance, and this constellation might not be automatized (i.e., people know how to recognize letters and how to push buttons; what they must learn is to push buttons in response to letters). In search tasks, then, the sets to discriminate and respond must be prepared and maintained by attending. Since preparation is the same for all array sizes, the amount of attention demanded should be independent of array size. However, the duration of attention demand should increase with array size: The set to discriminate and respond must be maintained until a response occurs, so the duration of attention demand will depend on reaction time. Since reaction time is determined by automatic letter-recognition processes whose duration 
increases with array size, the duration of attention demand depends, albeit indirectly, on the number of items in the array.

This interpretation illustrates a recent view of cognitive resource allocation that holds that the attention demands of performance are determined primarily by control processes that organize and coordinate the more elementary processes that actually deal with information (e.g., Logan, 1978; Newell, 1973; Posner \& Snyder, 1975). From this view, control processes organize the elementary processes in response to instructions or intentions to produce behavior appropriate to the situation. The elementary processes are relatively permanent residents of the mind and no longer require attention to be maintained. Their organization, however, is transitory, designed only to meet the needs of the moment, and so must be achieved and maintained by attending judiciously. A major empirical implication is that attention demands are determined by the control processes, not by the elementary processes they coordinate, nor even the information being processed. The present study, in finding no relation between attention demand and the amount to be processed (i.e., array size), is consistent with this general view. A major theoretical implication is that the problem of control must be taken seriously. Previous efforts have focused on processes in isolation or in some particular task environment. While this work is important, it ignores or deemphasizes the possibility that one process can serve many goals, and does little to specify the procedures by which processes are selected and combined to cope with new task environments. It is known from practical experience that human cognition is tremendously versatile; human subjects can perform virtually any task asked of them, often on a moment's notice. Perhaps by examining the processes of attentional control more directly than has been done in the past, psychologists will come to understand such versatility.

\section{REFERENCES}

Atrinson, R. C., Holmgren, J. E., \& Juola, J. F. Processing time as influenced by the number of elements in a visual display. Perception \& Psychophysics, 1969, 6, 321-326.

Comstock, E. M. Processing capacity in a letter-match task. Journal of Experimental Psychology, 1973, 100, 63-72.

Сомsтоск, Е. M.Limited-capacity central attention mechanisms. Unpublished $\mathrm{PhD}$ thesis, University of Massachusetts, 1975.

Exls, J. G. Analysis of temporal and attentional aspects of movement control. Journal of Experimental Psychology, 1973, 99, 10-21.

Estes, W. K. Interactions of signal and background variables in visual search. Perception \& Psychophysics, 1972, 12 , 278-286.

GARDNER, G. T. Evidence for independent channels in tachistoscopic perception. Cognitive Psycholosy. 1973, 4, 130-155.

Kanneman, D. Attention and effort. Englewood Cliffs, N.J: Prentice-Hall, 1973
Kantowitz, B. H. The double stimulation paradigm. In B. H. Kantowitz (Ed.), Human information processing: Tutorials in performance and cognition. Hillsdale, N.J: Lawrence Erlbaum, 1974.

KLAPP, S. T. Short-term memory as a response preparation state. Memory \& Cognition, 1976, 4, 721-729.

LOGAN. G. D. Converging evidence for automatic perceptual processing in visual search. Canadian Journal of Psychology, 1976, 30. 193-200.

LoGAN, G. D. Attention in character-classification tasks: Evidence for the automaticity of component stages. Journal of Experimental Psychology: General, 1978, 107, 32.63.

Millar, K. Processing capacity requirements of stimulus encoding. Acta Psychologica, 1975, 39, 393-410.

Newell, A. Production systems: Models of control structures. In W. G. Chase (Ed.), Visual information processing. New York: Academic Press, 1973.

Nickerson, R. S. Binary classification reaction time: A review of some studies of human information-processing capabilities. Psychonomic Monograph Supplements, 1972, 4, $275-318$

Posner, M. I., \& Bores, S. J. Components of attention. Psychological Review, 1971, 78, 391-408.

Posner, M. I., \& KleIN, R. On the functions of consciousness. In $\mathrm{S}$. Kornblum (Ed.), Attention and performance IV. New York: Academic Press, 1973.

Posner, M. I., \& SNyder, C. R. R. Attention and cognitive control. In R. Solso (Ed.), Information processing and cognition: The Loyola symposium. Potomac, Md: Lawrence Erlbaum, 1975.

Proctor, R. W., \& Fisicaro, S. A. Time, capacity, and selection between perceptual attributes. Journal of Experimental Psychology: Human Perception and Performance, 1977, 3, 337-348.

RUmelhaRT, D. E. A multicomponent theory of the perception of briefly exposed visual displays. Journal of Mathematical Psychology, 1970, 7, 191-218.

Shiffrin, R. M., \& Geisler, W. S. Visual recognition in a theory of information processing. In R. L. Solso (Ed.), Contemporary issues in cognitive psychology: The Loyola symposium. New York: Winston, 1973. Pp. 53-101.

ShIfFrin, R. M., \& SchneIder, W. Controlled and automatic human information processing: II. Perceptual learning, automatic attending, and a general theory. Psychological Review, 1977, 84, 127-190.

Shulman, H. G., \& Fisher, R. P. Expected value as a determinant of the distribution of attention. Journal of Experimental Psychology, 1972, 93, 343-348.

SHWARTZ, S. P. Capacity limitations in human information processing. Memory \& Cognition, 1976, 4, 763-768.

SMITH, E. E., \& SPOEkr, K. T. The perception of printed English: A theoretical perspective. In B. H. Kantowitz (Ed.), Human information processing: Tutorials in performance and cognition. Hillsdale, N.J: Lawrence Erlbaum, 1974.

STERnberg, S. Memory scanning: New findings and current controversies. Quarterly Journal of Experimental Psychology, 1975, 27, 1-32.

TAYlor, D. A. Stage analysis of reaction time. Psychological Bulletin, 1976, 83, 161-191.

TownSEND, J. T. Issues and models concerning the processing of a finite number of inputs. In B. H. Kantowitz (Ed.), Human information processing: Tutorials in performance and cognition. Hillsdale, N.J: Lawrence Erlbaum, 1974.

Vaughan, G. M.. \& Corballis, M. C. Beyond tests of significance: Estimating strength of effects in selected ANOVA designs. Psychological Bulletin, 1969, 72, 204-213.

(Received for publication November 15, 1977; accepted March 28, 1978.) 